\begin{tabular}{|c|l|}
\hline Title & Vortex tracking on visualized temperature fields in a rotating Ray leigh-Benard convection \\
\hline Author(s) & Noto, Daisuke; Tasaka, Y uji; Y anagisawa, Takatoshi; Park, Hyun Jin; Murai, Y uichi \\
\hline Citation & Journal of visualization, 21(6), 987-998 \\
https://doi.org/10.1007/312650-018-0510-6 \\
\hline Issue Date & 2018 12 \\
\hline Doc URL & http://hdl.handle.net/2115/76217 \\
\hline Rights & The original publication is available at www.springerlink.com \\
\hline Type & article (author version) \\
\hline File Information & NotoJoV 20180718c.pdf \\
\hline
\end{tabular}

Instructions for use 


\title{
Vortex tracking on visualized temperature fields in a rotating Rayleigh-Bénard convection
}

\author{
Daisuke Noto · Yuji Tasaka • Takatoshi \\ Yanagisawa • Hyun Jin Park • Yuichi \\ Murai
}

Received: / Revised: / Accepted:

\begin{abstract}
We established a vortex detection method using instantaneous temperature fields that were visualized using thermochromic liquid crystals (TLCs) to investigate behaviors of vortical structures in a rotating Rayleigh-Bénard convection. Experimental testing was performed at a fixed Rayleigh number $R a=$ $1.0 \times 10^{7}$, and different Taylor numbers from $T a=1.0 \times 10^{6}$ to $1.0 \times 10^{8}$ in water containing encapsulated TLCs. Vortices were recognized as undulations that appear in the horizontal temperature fields, thus making vortex detection with high spatial resolution possible, and this enabled quantitative investigation of the dynamics of vortical structures. Standard template matching was used to detect individual vortices on visualized temperature fields, and two-dimensional curved surface fitting was adopted to remove erroneous detections and to evaluate shapes of local temperature fields corresponding to vortical structures. Additionally, vortex tracking clearly showed geometric advection pattern of vortical structures.
\end{abstract}

Keywords Thermochromic liquid crystals · Rotating convection · Vortex tracking · Vortex behavior

\section{Introduction}

Rayleigh-Bénard convection (RBC) constrained by a background rotation has been investigated as one of the most fundamental systems in the study of fluid flows dominated by a thermal force and rotation. In particular, this system is recognized as an important model in the investigation of several aspects of geophysical fluid dynamics, including atmospheric flows, oceanic flows, and flows in the outer liquid core of the Earth. Typical RBC is a fluid flow confined by two parallel plates

D. Noto, Y. Tasaka, T. Yanagisawa, H. J. Park and Y. Murai

Laboratory for Flow Control, Faculty of Engineering, Hokkaido University, Sapporo, Japan

Tel.: +81-11-7066371

Fax: +81-11-7066373

E-mail: tasaka@eng.hokudai.ac.jp

T. Yanagisawa

Japan Agency for Marine-Earth Science and Technology (JAMSTEC), Yokosuka, Japan 


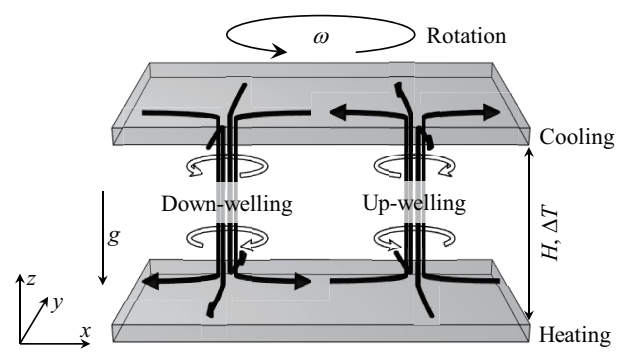

Fig. 1 Schematic diagram of quasi-2D vortical flow structures formed in rotating RBC

separated in the direction of gravity and has a temperature difference such that the top is cool and the bottom is hot. With the addition of background rotation, the flow can be organized by three dimensionless numbers. The Rayleigh number expresses the intensity of the thermal force given by $R a=g \beta \Delta T H^{3} /(\kappa \nu)$, where $g, \Delta T$, and $H$ are respectively the gravitational acceleration, the temperature difference between the top and bottom boundaries of the fluid layer, and the fluid layer height. Additionally, $\beta, \kappa$, and $\nu$ respectively denote the thermal expansion coefficient, thermal diffusivity, and kinematic viscosity of the working fluid. The Prandtl number is given as a physical property of the working fluid in the form $\operatorname{Pr}=\nu / \kappa$. The effect of the background rotation can be organized using the Taylor number and is given as the ratio of the Coriolis force to the viscous resistance, $T a=$ $\left(2 \omega H^{2} / \nu\right)^{2}$, where $\omega$ is the angular velocity of the rotating field. The convective Rossby number Ro or Ekman number $E$ has often been employed to describe the rotation intensity. These parameters are related according to

$$
R o=\frac{1}{2 \omega} \sqrt{\frac{g \beta \Delta T}{H}}=\sqrt{\frac{R a}{\operatorname{Pr} \cdot T a}}, E=\frac{\nu}{2 \omega H^{2}}=T a^{-1 / 2},
$$

where $T a$ is proportional to the square of the angular velocity $\omega$ and indicates the intensity of rotation directly.

In early stage of experimental studies on rotating RBC, optical visualization to observe vortical structures had been performed. As a typical characteristic of rotating RBC, quasi-two-dimensional (quasi-2D) flow structures form in regions where the fluid motions are strongly restricted by Taylor-Proudman theorem, as shown in Fig. 1. These vortical structures are stretched in the direction of the system rotation axis as the rotation rate increases. Vortical structures can be observed experimentally using flow visualizers, such as spherical particles, flake particles, and thermochromic liquid crystals (Cheng et al. (2015); Zhong et al. (1993); Sakai (1997); Kunnen et al. (2010)). Zhong et al. (1993) explained qualitative vortex behaviors including advection, generation, disappearance, and merging using visualized image sequences of vortical plumes at a timescale of $O(10-100 \mathrm{~s})$. This kind of vortex motion can be easily visualized, however, quantitative analysis or explanation have been avoided in previous researches. Investigation of long-timescale physics hidden behind rotating RBC is worthful and strongly required. Particle image velocimetry (PIV) is a well-known and sophisticated method for obtaining velocity fields; however, it is not suitable for multi-timescale parametric studies of this problem, because of problems of data storage and indirect vortex detections via calculating vorticity fields from the velocity fields. 
Encapsulated thermochromic liquid crystals (TLCs) can be used to visualize temperature fields; TLCs change their scattering/reflection characteristics for incident light with variations in the local temperature, and instantaneous temperature distributions appear as color variations on the visualized plane. In investigations of rotating RBC, Sakai (1997) visualized the temperature field using TLCs to confirm a theory of describing the horizontal scale of convection vortices, where complex advections of the vortices are represented by temporal changes in color patterns. Vorobieff and Ecke (1998) reported the evolution of vortical structures appearing during spin-up using TLCs. Zhou and Xia (2010) used TLCs to investigate sheetlike plumes appearing in RBC. Calibration between local coloration of TLCs and temperatures allows quantitative investigations of temperature fields. For vortex behaviors in rotating RBCs, the methodology has advantages of being grid free, allowing the setting of suitable time intervals, allowing the temperature to be given at each pixel, and having instantaneous single images unlike PIV. Additionally, under the condition $\operatorname{Pr} \geq O(1)$, vortex motions can be captured on temperature fields. In measurements of the temperature field using TLCs, however, multiple factors introducing uncertainties have been reported (Wiberg and Lior (2004); Rao and Zang (2010)), e.g., non-uniform incidence of illumination light, aging of TLCs, and etc. We thus have to consider the effect of relatively large uncertainties on measurements.

To investigate dynamical vortex behaviors, a method of detecting vortices from instantaneous temperature fields visualized by TLCs was established. To overcome relatively high uncertainty and noise in the temperature fields, a simple pattern recognition technique was applied to establish a highly versatile vortex detection method. This method is worthwhile as it can detect vortical structures as long as horizontal temperature fields are obtained. Thus, this vortex detection method is applicable over the flow regimes at which quasi-2D vortical structures are formed in both experimental and numerical results. Water $(\operatorname{Pr} \sim 7)$ was employed as the working fluid to ensure vortex tracking on temperature fields. Five different $T a$ conditions with fixed $R a$ were experimentally examined to clarify the development of vortex behaviors. Vortex tracking was performed by analyzing consecutive visualized images to reveal dynamics of the vortical structures.

\section{Experimental setup}

The experiment was conducted in a cylindrical fluid layer as illustrated in Fig. 2. The fluid layer was prepared by insertion of an acrylic annulus with inner diameter $D$ of $190 \mathrm{~mm}$, height $H$ of $40 \mathrm{~mm}$, and $5 \mathrm{~mm}$ lateral wall thickness into $40-\mathrm{mm}-$ height square vessel with 200 -mm-long sides. The aspect ratio of the fluid layer here is given as $\Gamma=4.75$. Note that this aspect ratio may produce a centrifugal force effect on the vortex behaviors. A brief calculation of the centrifugal force contribution is given as Froude number, centrifugal force/gravity force, $F r=D \omega^{2} /(2 g)$. In this system, $F r \sim 0.1$ at the largest rotation rate, namely, the centrifugal force has an impact of up to $10 \%$ when compare with the gravitational force in the vicinity of the side wall $(D / 2=95 \mathrm{~mm})$. The bottom of the vessel was heated using a copper plate heater with a thin (less than 0.01-mm-thick) black paint coating for visualizations; its temperature was monitored constantly using a thermistor probe embedded in the plate and a constant temperature was maintained by controlling 

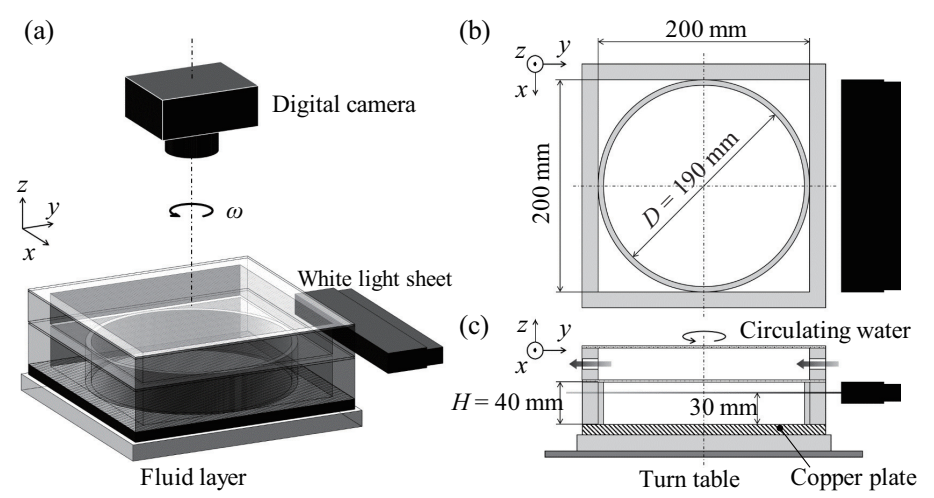

Fig. 2 Experimental setup; (a) overview, (b) top view, and (c) side view

the electric power supplied to the heater. The top was cooled using a thermostatic layer filled with circulating cool water from a thermostatic bath. Heat transfer from the top was conducted through the glass plate and its transparency allowed picture to be taken from above the fluid layer. For the same purpose, Kunnen (2008) employed a thin Plexiglas with $1 \mathrm{~mm}$ thickness. With considering that thermal conductivity of Plexiglas is approximately one third or fourth lower than that of glass, 3-mm-thick of the present glass plate provides similar quality for the heat transfer with the work. Corresponding Biot number of the top plate is round 0.6. But, thanks to the thickness of the glass plate, homogeneous heat conduction is expected. Preliminary measurement of surface temperature on the plate by multiple thermistors sufficiently high uniformity of the temperature within resolution of the thermistor, around $0.1^{\circ} \mathrm{C}$.

Degassed water containing suspended microcapsules of TLCs with $0.04 \mathrm{wt} \%$, which can visualize temperatures noninvasively in fluids as colors, was used as the working fluid. The TLCs change their scattering/reflection characteristics for incident light with variations in local temperature, and instantaneous temperature distributions appear as color variations on the visualized plane. The TLC colors vary from red to blue, corresponding to low to high temperature. Note that this is the reverse of the conventional colors used for human recognition. Specifications of encapsulated TLCs used in this study are $10-20 \mu \mathrm{m}$ diameter, 1.01 specific gravity, and 24.5 to $27^{\circ} \mathrm{C}$ of thermochromic range (Japan capsular products inc.). Corresponding Stokes number is $O\left(10^{-6}\right)$, where Prandtl's free-fall velocity, $O\left(10^{-2}\right.$ $\mathrm{m} / \mathrm{s}$ ) in the present system, is adopted as the representative velocity. Hence TLCs behave as ideal tracers of the flows. Thermochromic range of TLCs was chosen to cover the bulk temperature range to visualize whole horizontal temperature fields. To achieve flow field visualization, a white light sheet from a halogen lamp with minimum thickness of $2 \mathrm{~mm}$ illuminated the horizontal plane at a height of $30 \mathrm{~mm}$ $(z=0.75 H)$ from the bottom.

Background rotation of the system was performed by installing the fluid layer on a turn-table composed of two stages. The round tables consist of 1-m-diameter stainless steel plates which offer sufficient space to arrange all the experimental equipment. On the first plate, the fluid layer, a thermistor probe, and the white light sheet generator are mounted. On the second plate, the digital camera and 
Table 1 Specifications of the experimental conditions ( $R a$ and $T a$ ); theoretically estimated scales: thickness of thermal and Eckman boundary layers ( $\delta_{\mathrm{t}}$ and $\delta_{\mathrm{E}}$ ), horizontal scale of vortical structures $(L)$, and vortex radius $\left(R_{\mathrm{V}}\right)$; the number of up-welling and down-welling vortical structures detected from temperature field at $z=0.75 \mathrm{H}$

\begin{tabular}{|c|c|c|c|c|c|c|c|c|c|}
\hline \multirow{2}{*}{$R a$} & \multirow{2}{*}{$T a$} & \multirow{2}{*}{$\delta_{\mathrm{t}}[\mathrm{mm}]$} & \multirow{2}{*}{$\delta_{\mathrm{E}}[\mathrm{mm}]$} & \multirow{2}{*}{$L[\mathrm{~mm}]$} & \multirow{2}{*}{$R_{\mathrm{V}}[$ pixel] } & \multicolumn{2}{|c|}{ Up-welling } & \multicolumn{2}{|c|}{ Down-welling } \\
\hline & & & & & & Average & Std. dev. & Average & Std. dev. \\
\hline \multirow{5}{*}{$1.0 \times 10^{7}$} & $1.0 \times 10^{6}$ & \multirow{5}{*}{0.706} & 1.79 & 16.5 & 61 & 16.0 & 3.04 & 52.6 & 6.16 \\
\hline & $3.0 \times 10^{6}$ & & 1.36 & 15.4 & 57 & 21.9 & 3.13 & 53.7 & 5.68 \\
\hline & $1.0 \times 10^{7}$ & & 1.01 & 13.7 & 50 & 74.7 & 5.92 & 114.2 & 7.19 \\
\hline & $3.0 \times 10^{7}$ & & 0.76 & 11.7 & 43 & 93.0 & 5.21 & 122.8 & 6.44 \\
\hline & $1.0 \times 10^{8}$ & & 0.57 & 9.3 & 34 & 198.8 & 8.41 & 214.2 & 7.82 \\
\hline
\end{tabular}

two laptop computers to control the electric heater and the camera are mounted. Additionally, the second plate has a central hole to enable setting of a digital camera to photograph the illuminated fluid layer when fixed at the center of the first plate. The visualized images were recorded in a 24-bitmap format, in which the red, green and blue imaging elements have 8-bit levels set by the camera. Table rotation was controlled using an AC servo motor connected to a table rotation shaft by a belt. The angular velocity $\omega$ was adjustable over the $0.0-5.0 \mathrm{rad} / \mathrm{s}$ range (with corresponding $T a$ numbers of $0-2.6 \times 10^{8}$ ). Using the rotary joint connected to the shaft, cooling water and electricity for the experimental devices can be supplied to the table from the exterior, even during rotation. The digital camera and other electrical devices can be operated by remote control communicating via a wireless local area network (LAN) through a laptop computer located outside the rotating table.

\section{Temperature fields and vortices}

\subsection{Visualized temperature field}

Flow visualization using the TLCs was performed by interval photography. Photographic conditions including a frame rate of $1 / 3 \mathrm{fps}$, f-number of 2.7 , and resolution of $0.1357 \mathrm{~mm} /$ pixel were chosen for optimal visualization. Experimental conditions are summarized in Table 1 . All the experiments were performed at fixed $\operatorname{Pr}$ and $R a, \operatorname{Pr}=7$ and $R a=1.0 \times 10^{7}$ (corresponding temperature difference is $\Delta T=11.1^{\circ} \mathrm{C}$ : top $16.4^{\circ} \mathrm{C}$ and bottom $27.5^{\circ} \mathrm{C}$ ), in order to use the same TLCs for different conditions. Some papers reported that quasi-2D vortex structures are observed even for smaller $\mathrm{Ta}$ conditions, $10^{5}<\mathrm{Ta}<10^{7}$ (e.g. Zhong et al. (1993); Sakai (1997); Vorobieff and Ecke (2002)), and thus expected flow states should be quasi-2D, which can be observed well in horizontal cross-sections. In particular, down-welling vortices are clearly visualized because the height of visualized crosssections is always fixed at $z=0.75 H$. Thanks to vertically symmetrical systems, only upper side visualization is sufficient to know the flow structures. Examples of visualized temperature field images are shown in Fig. 3. These images were taken after the flow fields had reached equilibrium states (more than one hour after starting rotations). Vortical structures with relatively high temperatures are visualized as blue circles, with those at lower temperatures shown as red circles, 
and are dependent on the TLCs characteristics. At $T a=1.0 \times 10^{6}$ and $3.0 \times 10^{6}$ cases, some vortical structures can be observed although the fluid layer is not fully occupied by the vortical structures and some margin, where no vortex exists, are standing out compared to other rotational conditions. In our observations, these conditions cannot succeed to form vortical structures for a long time, mainly less than seconds, $10 \mathrm{~s}$ as the longest. This suggests the vortical structures are not strong and more like thermal plumes in these conditions. For comparison to other conditions, these conditions are also analyzed later. The fluid layer is horizontally filled with vortical plumes in the cases of $T a \geq 1.0 \times 10^{7}$. Apparently, these conditions successfully formed quasi-2D vortical structures. Moreover, the interesting thing is clear in the case of $T a=1.0 \times 10^{8}$. Relatively round and isolated vortical structures are observed entire the fluid layer except within the vicinity of the wall. What should be pointed out here is the number and the size of vortical structures. Obviously, the number of vortical structures increases according as $T a$ increases. As if it is inversely proportional to this, the size becomes smaller according as $T a$ increases. In these raw images, it is easy for human's eye to recognize vortical structures while actual temperatures are still unknown. There is a radial coloration trend observed only at $T a=1.0 \times 10^{8}$. This will be discussed later with the temperature conversion.

\subsection{Temperature conversion}

As described above, the colors of the TLCs are recorded in 24-bitmap format, which includes red-green-blue (RGB) information in each pixel. Several TLC color-totemperature calibration methods have been proposed, including hue calibration and use of neural network (Abdullah et al. (2010); Dabiri and Gharib (1991); Fujisawa et al. (2005); Ahmed et al. (1997); Park et al. (2001)). In the present study, the uniform surface temperature method was employed. In this method, fluid temperatures were fixed at predefined constant values for the response time of the TLCs or more. The temperatures were set at increment of $0.1^{\circ} \mathrm{C}$ from $24.5^{\circ} \mathrm{C}$ to $27.0^{\circ} \mathrm{C}$ and the reference color information can be acquired by photographing. An example of coloring appearance at each temperature is shown in Fig. 4(a). In the hue calibration method used here, RGB temperature information is converted to the hue, saturation and intensity (HSI) color space, which is a type of color appearance system. The following biconic HSI model is used here because of its simplicity.

hue $= \begin{cases}60 \times \frac{G-R}{\max (R, G, B)-\min (R, G, B)}+60, & \text { if } \min (R, G, B)=B \\ 60 \times \frac{B-G}{\max (R, G, B)-\min (R, G, B)}+180, & \text { if } \min (R, G, B)=R \\ 60 \times \frac{R-B}{\max (R, G, B)-\min (R, G, B)}+300, & \text { if } \min (R, G, B)=G \\ \text { undefined, } & \text { if } \min (R, G, B)=\max (R, G, B)\end{cases}$

Here, $R, G$, and $B$ are RGB information values recorded in a range from 0 to 255 , i.e., in 8-bit levels. The function, $\max (R, G, B)$ and $\min (R, G, B)$ are the maximum and minimum RGB values at each pixel. Hue values vary from 0 to 360 degrees, with zero corresponding to red and 180 to blue-green, which is the opposite color 

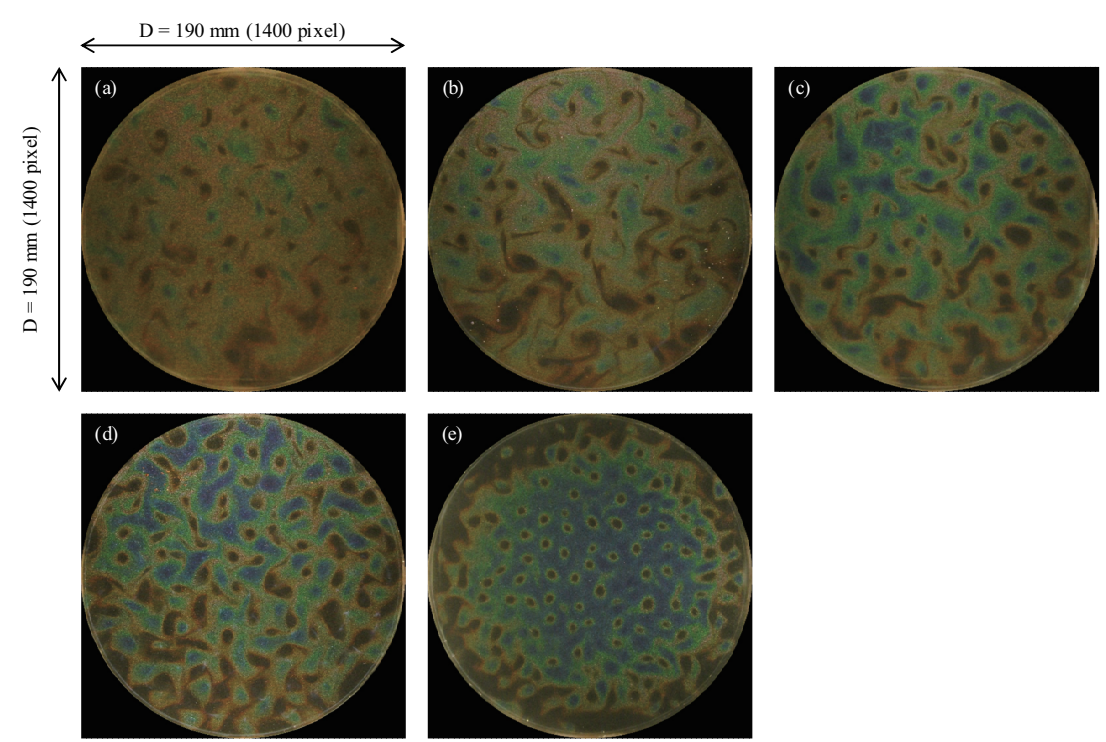

Fig. 3 Examples of visualized temperature fields (a) $T a=1.0 \times 10^{6}$, (b) $T a=3.0 \times 10^{6}$, (c) $T a=1.0 \times 10^{7}$, (d) $T a=3.0 \times 10^{7}$, and (e) $T a=1.0 \times 10^{8}$, where white light sheet illuminated the fluid layer from upper side of the pictures.

to red in the color circle. TLCs are highly sensitive to incident light and their color appearances are strongly dependent on the attenuation of the light sheet, the observation direction (the angle measured from the lens), reflections at the cylindrical wall in the present case, and coloring changes that are dependent on location in the images. To relieve these optical sensitivity effects, a split calibration method is proposed in which a visualized image is divided into multiple subregions and the calibration curves are calculated individually in each small region. Here, the entire fluid layer $(1400 \times 1400$ pixels $)$ is divided into $400(=20 \times 20)$ square subregions; each region thus has an area of $70 \times 70$ pixels corresponding to $9.5 \mathrm{~mm}$ $\times 9.5 \mathrm{~mm}$. Spatially averaged hue value in each subregion is used for temperature calibration to third-order accuracy using the least-squares method. An example of calibration curve is shown in Fig. 4(b), where the error bars indicate standard deviation of hue value in the subrange. Relatively large deviations appear for temperatures larger than $26^{\circ} \mathrm{C}$ and are caused by irregular, spiky scatters of hue values. In the analysis, such spiky noise will be removed by median filtering that was also used to overcome general noise arising from TLCs (Wiberg and Lior (2004); Rao and Zang (2010)) as detailed in the sections below.

There are non-negligible uncertainties, particularly in the range of higher temperature. Such uncertainties, however, do not affect vortex detection explained in the next section. The results of RGB to hue conversion of the raw images are shown in Fig. 5(a), (b), and (c) that are the representative examples at Ta $=3.0 \times 10^{6}$, $3.0 \times 10^{7}$, and $1.0 \times 10^{8}$. Thanks to these hue conversion and temperature calibration, in the figures, two types of vortical structures can easily be distinguished because color information is expressed using continuous hue and temperature values. This helps to deal characteristic values expressing vortical structures by a single value, hue or temperature. Temperature fields produced by conversion us- 
(a)

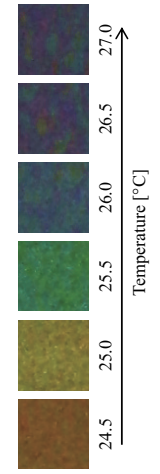

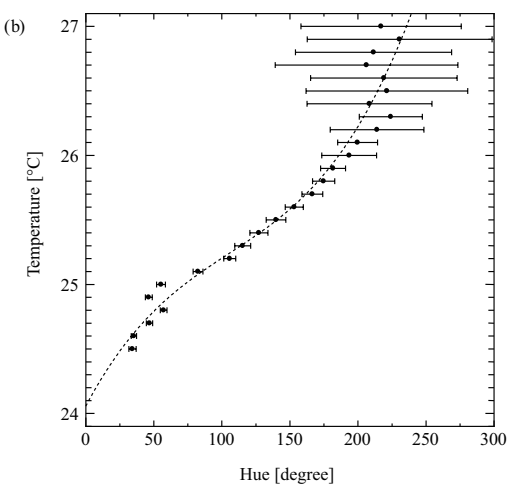

Fig. 4 Example of (a) image series of reference colors and (b) calibration curve between temperature and hue
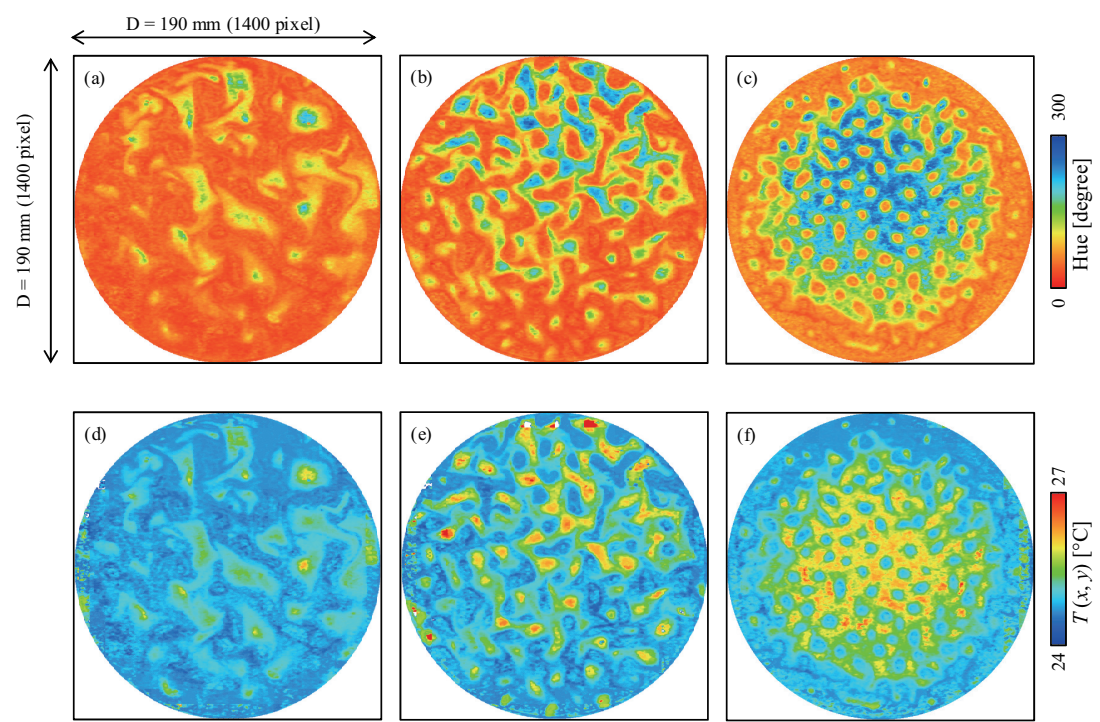

Fig. 5 Examples of hue distributions converted from visualized images taken at (a) $T a=$ $3.0 \times 10^{6}$, (b) $3.0 \times 10^{7}$, and (c) $1.0 \times 10^{8}$; (d)-(f) horizontal temperature fields obtained from hue distribution, (a)-(c) respectively, using the calibration curve

ing the calibration curves from the hue distribution in Fig. 5(a), (b), and (c) are shown in Fig. 5(d), (e), and (f), respectively.

The split calibration method can eliminate color trend caused by the optical sensitivity of TLCs. A temperature trend, however, certainly exists in the radial direction as shown in Fig. $5(\mathrm{f})$ at $T a=1.0 \times 10^{8}$. As the reason, centrifugal force, imperfections of the setup, influence of side-wall boundary layer are candidates. Thanks to the water jacket around the annulus, unignorable temperature difference between inside and outside should not exist. And, test numerical simulations excluding centrifugal term performed ourselves do not show any radial trend on the temperature field. The centrifugal force effect is therefore expected from this image. On the contrary, there is no such certain trend at $T a=3.0 \times 10^{6}$ and $3.0 \times 10^{7}$ 


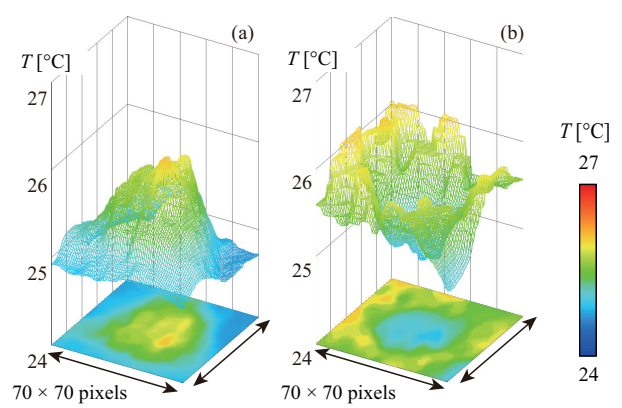

Fig. 6 Examples of temperature distributions inside (a) up-welling and (b) down-welling vortices at $T a=1.0 \times 10^{8}$

(Fig. 5(d) and (e)); the effect of centrifugal force represented by Froude number is $F r=D \omega^{2} /(2 g) \sim 0.1$ at $T a=1.0 \times 10^{8}$, while $F r \sim 0.003$ at $T a=3.0 \times 10^{6}$.

\subsection{Vortices recognized on the temperature fields}

There is a great deal of unevenness in the horizontal temperature fields shown as examples in Fig. 5(d), (e), and (f). When the temperature fields are compared to the visualized image in Fig. 3, this unevenness represents a vortex; concave features correspond to down-welling vortices while convex features represent up-welling vortices. Small-scale fluctuations also exist on the concave and convex shapes that result from the uncertainty of the temperature calibration from the visualized images. We used median filtering with the window size of $3 \times 3$ pixels, only to eliminate noises caused by dust or bubbles appeared during experiments, not to relieve small-scale fluctuations. Hence, all the analyses afterward are performed while such inevitable small-scale fluctuations remain.

Stevens et al. (2012) reported good agreement of temperature isosurfaces with $Q_{3 \mathrm{D}}$ criterion-based detection of vortices in their numerical simulations $(\mathrm{Pr}=$ 4.38). Examples of up-welling and down-welling vortices extracted individually from Fig. 5(f) are shown in Fig. 6(a) and (b), respectively. The diameters of both the up-welling and down-welling vortices at $\mathrm{Ta}=1.0 \times 10^{8}$ are approximately $9.5 \mathrm{~mm}$ (= 70 pixels) in Fig. 6(a) and (b). In the up-welling vortex shown in Fig. 6(a), the temperature inside the vortex gradually increases toward the center, while a contrasting trend is observed in the down-welling vortex in Fig. 6(b). Such characteristic trend is not buried by small-scale fluctuations mentioned above. According to these observations, the vortex outline can be extracted using a relatively simple image processing technique that judges whether the area is convex or concave.

\section{Vortex detection}

The method composes three steps: Gaussian template matching, 2D curved surface fitting, and error elimination. First, Gaussian template matching approximates the vortex coordinates as an application of an image processing technique. Second, 2D 
curved surface fitting measures the detected vortex coordinates more precisely and categorizes vortices using their interior temperature distributions. Finally, an error elimination method that considers vortex dynamics is proposed. These processes are explained in detail and discussed below.

\subsection{Gaussian template matching}

Gaussian template matching has been used to detect, for example, tracer particles for PIV. Seeking objects on images are recognized as similar areas to those of the template by calculating a cross-correlation coefficient. Here, distinctive temperature distributions are observed inside vortices, such as the temperature increasing towards the center in up-welling vortices but decreasing towards the center in down-welling vortices, as described above, and these temperature distributions are appropriate for template matching. Sakai (1997) derived the horizontal scale of convection cells in a rotating $\mathrm{RBC}$, and described it as

$$
L=4.6 H \frac{\delta_{\mathrm{t}}^{2}}{2 \delta_{\mathrm{t}}^{2}+2 \delta_{\mathrm{t}} \delta_{\mathrm{E}}+\delta_{\mathrm{E}}^{2}} R a^{1 / 3} T a^{-3 / 8}
$$

where $L$ is the horizontal convection cell scale, and $\delta_{\mathrm{E}}$ and $\delta_{\mathrm{t}}$ are the thicknesses of the Ekman layer and the thermal boundary layer, respectively:

$$
\begin{aligned}
\delta_{\mathrm{E}} & =\sqrt{2} H T a^{-1 / 4}, \\
\delta_{\mathrm{t}} & =3.8 H R a^{-1 / 3} .
\end{aligned}
$$

This theoretically estimated scale agreed reasonably well with the experimental values in Sakai (1997) and those in the present experiments. From this scale, the estimated vortex radius $R_{\mathrm{v}}$ with units of pixels in the present case can be calculated as $2 R_{\mathrm{v}}=1400 L / 190$. Here, $R_{\mathrm{v}}$ estimated for all the experimental parameters are summarized in Table $1 . R_{\mathrm{v}}$ at $T a=1.0 \times 10^{8}$, for instance, is approximately 34 pixels and this demonstrates that the horizontal scale shown in Fig. 6 (=70 pixels) looks plausible to express the vortical structures. Note that the vortex temperature distributions are either concave or convex and can be regarded as point-spread shapes. Using the vortex radius calculated above, the Gaussian template is created accordingly as

$$
G(x, y)=\frac{1}{\sqrt{2 \pi \sigma^{2}}} \exp \left(-\frac{x^{2}+y^{2}}{2 \sigma^{2}}\right), \sigma=R_{\mathrm{v}} / 2
$$

Size of the template is variable in pattern matching, and setting such a reference value as the scale can reduce erroneous detections and calculation costs. Horizontal cross-sections of vortices are not perfect round as the template, but elliptical shape, because of mutual interactions between surroundings. The Gaussian template matching therefore seeks undulations around the centers of vortices at which most cold or hot region exist.

With taking account fine undulations in a single vortex, positions with remarkable cross-correlation coefficients when compared with the twenty-four pixels surrounding them $(=5 \times 5$ pixels $)$ are enumerated as candidates of the vortex center. When a convex upward Gaussian distribution is used, positive correlation 
areas are thought to be up-welling vortices while negative correlation areas are down-welling vortices. The threshold should therefore be set at both positive and negative values to detect both up-welling and down-welling vortices with a single scan. Taking these factors into account, the thresholds of the cross-correlation values are set at \pm 0.5 for Gaussian template matching.

\section{$4.22 \mathrm{D}$ curved surface fitting}

Gaussian template matching cannot detect all the vortices precisely for two reasons. One reason is the considerable deviation of the temperature distributions inside the vortices from the ideal Gaussian template. As shown in Fig. 4, actual temperature distributions inside vortices are not smooth like a Gaussian distribution, and these deviations increase because of interactions between vortices. Here, 2D curved surface fitting (Sheng et al. (2001); Hyyppä et al. (2001)) is proposed as one error elimination method, along with a more precise rearrangement of the detected position.

According to Sheng et al. (2001), a 2D curved surface for the fitting is given as the general ellipsoid equation

$$
\frac{z^{c}}{h^{c}}+\frac{\left(x^{2}+y^{2}\right)^{\frac{c}{2}}}{r^{c}}=1
$$

where $r, h$, and $c$ are the radius, the height, and the curvature, respectively. In the present use, $r$ and $h$ are replaced by radius of the vortices $R_{\mathrm{f}}$ and the temperature difference between the vortex center and its edge, $H_{\mathrm{f}}$. Here, $R_{\mathrm{f}}, H_{\mathrm{f}}$ and $c$ are variable in ranges, 30 to 38 pix with two pixels as increment, every $0.15^{\circ} \mathrm{C}$ from 0.30 to $0.75^{\circ} \mathrm{C}$, and every 0.25 from 0.75 to 1.75 . By combining these parameters, all vortices are categorized into $100(=5 \times 4 \times 5)$ patterns. The fitting with modifying the parameters is performed around the central position coordinates of vortices determined by the Gaussian template matching in a surrounding area with $7 \times 7$ pixels. The position giving better $2 \mathrm{D}$ curved surface fitting is thus used as the center of vortices. In cases that the suitable fitting is not provided, the original positions are recognized as erroneous detections of vortices and thus are eliminated.

The advantages of this $2 \mathrm{D}$ curved surface fitting method include high accuracy detection of the vortex center position and ability to categorize vortices into several patterns based on their temperature distribution shapes. In this study, these vortices are categorized into 100 patterns using their radius, height, and curvature features, and this information can be used for quantitative evaluation of vortex behavior from a heat transfer viewpoint. Additionally, by evaluating the category transitions with time, the vortex shape transitions in horizontal planes can be evaluated.

\subsection{Recognizing merging vortices and error elimination}

To detect vortices automatically, Gaussian template matching and 2D curved surface fitting method have been proposed thus far. In the processing of these two 


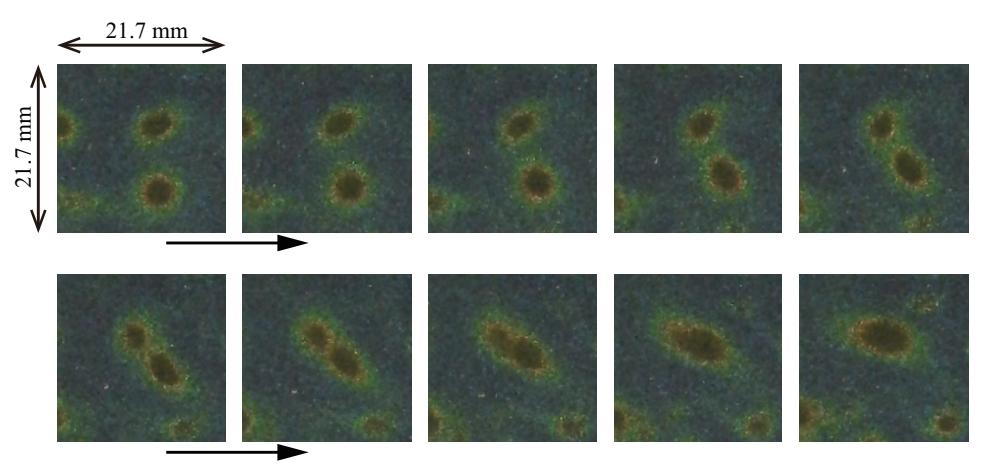

Fig. 7 Sequential temperature field images at intervals of $3 \mathrm{~s}$ representing two merging vortices

methods, however, it is possible that erroneous detections such as overlap detection for single vortices remain. To eliminate these errors, a judgment criterion is required to determine whether the vortex is erroneous or correct. This criterion should not affect subsequent quantitative analyses like vortex tracking. To satisfy this condition, one characteristic phenomenon that is considered is merging of multiple vortices. As shown by sequential images of the temperature field in Fig. 7, merging of vortices is often observed in actual flow fields. All vortices move around during the period from generation to disappearance or merging. Merging of multiple vortices is represented as approaching and following duplication of the centers of vortices. Near the end of the merging process, two vortex centers exist reasonably closely together and such cases must be excluded from overlapping detections to prevent recognition of erroneous detections as correct detections. In this method, if a vortex center exists inside another vortex area determined by a circle with fitted radius $R_{\mathrm{f}}$, it is regarded as a merging event and the higher fitted vortex is preserved by comparing the determination coefficients calculated during 2D curved surface fitting. By repeating these processes until all the overlaps are solved, all Gaussian template matching errors can be eliminated.

\subsection{Results}

An example result of use of all the proposed procedures, including Gaussian template matching, 2D curved surface fitting, and error elimination while considering merging vortices, is shown in Fig. 8(a), where up-welling and down-welling vortices are indicated using different symbols. The vortices are distributed over the entire fluid layer, and there are no clear nonuniformities in the distributions of the up-welling and down-welling vortices despite difference on visibility between them in the temperature field (see Fig. 5). The improvement in vortex detection accuracy through the proposed processes is shown from Fig. 8(b) to (d). Original detection results by Gaussian template matching shown in Fig. 8(b) contains many erroneous detections represented as unusually close positions of the vortices. These are improved after taking 2D curved surface fitting (Fig. 8(c)), although there are still some overlaps of the vortex centers. Through all the procedures, in Fig. 8(d), the overlaps are resolved and individual vortices can be distinguished easily. The number of detected vortical structures for one hour after reached equi- 


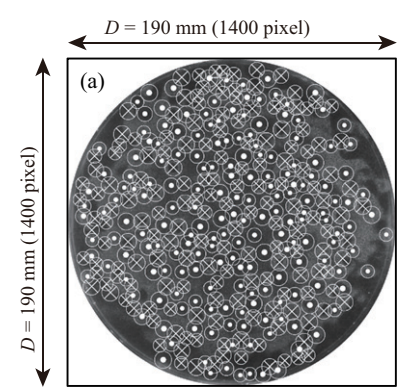

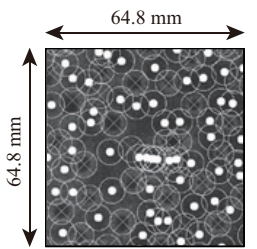

(b)

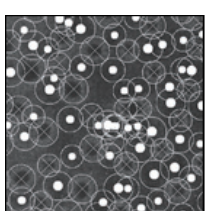

(c)

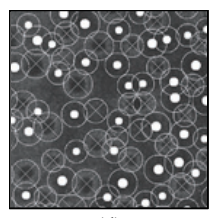

(d)

Fig. 8 Examples of detected vortices plotted on the original visualized temperature field image. (a) Result for the entire fluid layer for all processes, and enlarged views after (b) Gaussian template matching, (c) 2D curved surface fitting and (d) error elimination over the same area

librium states are summarized in Table 1. Difference between the numbers of detected up-welling and down-welling vortical structures decreases as increase of $\mathrm{Ta}$. This is reasonable because our observation cross-section is at $z=0.75 \mathrm{H}$ and this yields prominent existence of down-welling vortices. Pieri et al. (2016) showed the vertical variations of the number of vortical structures at a horizontal plane. The present results quantitatively agree with the result at the same height shown by Pieri et al. (2016). We can perform this series of procedures on all instantaneous temperature fields, and detected and labeled vortices enable investigation of vortex behaviors in rotating RBCs.

The path-lines of vortex centers obtained from 30 sequential images of temperature fields taken over $90 \mathrm{~s}$ at intervals of $3 \mathrm{~s}$ are discussed in terms of the advection characteristics in $O(10 \mathrm{~s})$ (Fig. 9). Up-welling and down-welling vortices are plotted using circles and diagonal crosses in the figure. There are no specific patterns in Fig. 9(a) and the distributions of plots seem to be disorganized. On the contrary, each path-line drawn by individual single vortical structures can be recognized at $\mathrm{Ta}=3.0 \times 10^{7}$ (Fig. 9(b)). Their advections from generation to disappearance or merging are completed inside the cropped section shown in the figure. The organized path-lines in Fig. 9(b) indicate organized behaviors of vortical structures stabilized in the vertical direction. An interesting finding can be seen in Fig. 9(c); although the number of images is reduced from the original sequence and thus there are blanks between plots, geometric patterns of the path-lines are clearly observed. Up-welling vortices move around a down-welling vortex, and the corresponding path-lines form quasi-polygonal shapes or at least, any organized pattern. These geometric structures have not been mentioned in previous studies. Also, their path-lines are not completed inside the cropped section shown in Fig. 9(c). A brief review of the geometric patterns as understood from the visualized image indicates that the vortex arrangement has some regularity in this $O(10 \mathrm{~s})$ timescale. All vortices continue advection while maintaining the distances between adjacent vortices, i.e., the advection is regarded as an $n$ body problem. While detailed discussions of the geometric patterns of advection are avoided in this paper, these patterns seem to be formed by the mutual interactions of vortices. As a reference for the considerations, timescale of the system rotation $\tau_{R}=2 \pi / \omega=1.26 \mathrm{~s}$ is much shorter than the timescale for observing above-mentioned patterns. 

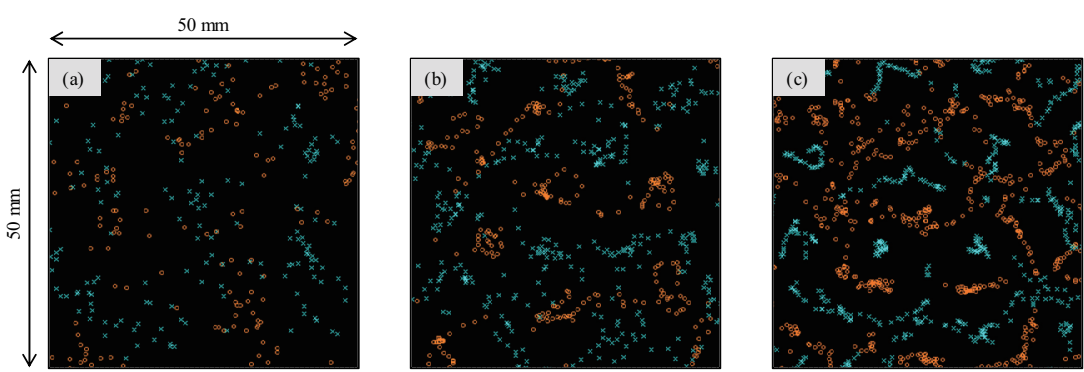

Up-welling vortex $\times$ Down-welling vortex

Fig. 9 Examples of path-lines of quasi-2D vortical structures for $90 \mathrm{~s}$ obtained by plotting detected vortex positions at (a) $T a=3.0 \times 10^{6}$, (b) $3.0 \times 10^{7}$, and (c) $1.0 \times 10^{8}$

\section{Conclusions}

To reveal the dynamics of vortical structures appeared in rotating RayleighBénard convection, we have established a vortex detection method in cross-sections based on images of instantaneous horizontal temperature fields visualized using thermochromic liquid crystals. Undulations in the temperature field expressing the vortices were detected using template matching, and the detected vortex coordinates were improved by classifying the temperature distribution shapes inside the vortices into several patterns described as ellipsoids. The latter treatment also enables vortex classification from a temperature distribution shape perspective. In addition, by combining an error elimination method with consideration of merging vortices, the vortex advection may become experimentally trackable. Advantage of the present method is a high versatility for wide range of rotational parameters at which quasi-2D vortex structures are distinguished as undulations on horizontal temperature fields, thanks to multiple, but simple detection algorithms. Applying the method to the actual flow field elucidated the typical advection characteristics of the vortical structures; Over the timescale $O(10 \mathrm{~s})$, geometric advection patterns were formed at the highest rotational condition.

\section{References}

Abdullah N, Talib ARA, Jaafar AA, Salleh MAM, Chonga WT (2010) The basics and issues of Thermochromic Liquid Crystal Calibrations. Exp Therm Fluid Sci 34:1089-1121

Ahmed S, Russell P, Lisboa P, Jones GR (1997) Parameter monitoring using neural-networkprocessed chromaticity. IEE P-Sci Meas Tech 144:257-262

Cheng J, Stellmach S, Ribeiro A, Grannan A, King E, Aurnou J (2015) Laboratory-numerical models of rapidly rotating convection in planetary cores. Geophys J Int 201:1-17

Dabiri D, Gharib M (1991) Digital particle image thermometry: The method and implementation. Exp Fluids 11:77-86

Fujisawa N, Futamaki S, Katoh N (2005) Scanning liquid-crystal thermometry and stereo velocimetry for simultaneous three-dimensional measurement of temperature and velocity field in a turbulent Rayleigh-Bérnard convection. Exp Fluids 38:291-303

Hyyppä J, Kelle O, Lehikoinen M, Inkinen M (2001) A segmentation-based method to retrieve stem volume estimates from 3-D tree height models produced by laser scanners, IEEE T. Geosci. Remote 39:969-975

Kunnen RPJ (2008) Turbulent rotating convection, Ph.D. thesis, Technische Universiteit Eindhoven. 
Kunnen RPJ, Clercx HJH, Geurts BJ (2010) Vortex statics in turbulent rotating convection. Phys Rev E 82:036306

Park H, Dabiri D, Gharib M (2001) Digital particle image velocimetry/thermometry and application to the wake of a heated circular cylinder. Exp Fluids 30:327-338

Pieri AB, Falasca F, Hardenberg JV, Provenzale A (2016) Plume dynamics in rotating Rayleigh-Bénard convection. Phys Lett A 380:1363-1367

Rao Y, Zang S (2010) Calibrations and the measurement uncertainty of wide-band liquid crystal thermography. Meas Sci Technol 21:015105

Sakai S (1997) The horizontal scale of rotating convection in the geostrophic regime. J Fluid Mech 333:85-95

Sheng Y, Gong P, Biging GS (2001) Model-Based Conifer-Crown Surface Reconstruct ion from High-Resolution Aerial Images. Photogramm Eng Remote Sensing 8:957-965

Stevens RJAM, Clercx HJH, Lohse D (2012) Breakdown of the large-scale wind in $\Gamma=1 / 2$ rotating Rayleigh-Bénard flow. Phys Rev E 86:056311

Vorobieff P, Ecke RE (1998) Vortex structure in rotating Rayleigh-Bénard convection. Physica D 123:153-160

Vorobieff P, Ecke RE (2002) Turbulent rotating convection: an experimental study. J Fluid Mech 458:191-218

Wiberg R, Lior N (2004) Errors in thermochromic liquid crystal thermometry. Rev Sci Instrum $75: 2985$

Zhong F, Ecke RE, Steinberg V (1993) Rotating Rayleigh-Bénard convection: asymmetric modes and vortex states. J Fluid Mech 249:135-159

Zhou Q, Xia K (2010) Physical and geometrical properties of thermal plumes in turbulent Rayleigh-Bénard convection. New J Phys 12:075006 